\title{
Model-based three-dimensional texture analysis of contrast-enhanced magnetic resonance imaging as a potential tool for preoperative prediction of microvascular invasion in hepatocellular carcinoma
}

\author{
YONG-JIAN ZHU ${ }^{1}$, BING FENG $^{1}$, SHUANG WANG ${ }^{1}$, LI-MING WANG ${ }^{2}$, \\ JIANG-FEN WU ${ }^{3}$, XIAO-HONG MA ${ }^{1 *}$ and XIN-MING ZHAO $^{1 *}$ \\ Departments of ${ }^{1}$ Imaging Diagnosis and ${ }^{2}$ Hepatobiliary Surgery, \\ National Cancer Center/National Clinical Research Center for Cancer/Cancer Hospital, \\ Chinese Academy of Medical Sciences and Peking Union Medical College, Beijing 100021; \\ ${ }^{3}$ GE Healthcare Shanghai Co., Ltd., Shanghai 201203, P.R. China
}

Received June 24, 2018; Accepted April 15, 2019

DOI: $10.3892 / 01.2019 .10378$

\begin{abstract}
The purpose of the present study was to investigate the value of contrast-enhanced magnetic resonance imaging (CE-MRI) texture analysis for preoperatively predicting microvascular invasion (MVI) in hepatocellular carcinoma (HCC). Accordingly, a retrospective study of 142 patients with pathologically confirmed HCC was performed. The patients were divided into two cohorts: The training cohort $(n=99)$ and the validation cohort $(n=43)$, including the MVI-positive
\end{abstract}

Correspondence to: Professor Xiao-Hong Ma or Professor Xin-Ming Zhao, Department of Imaging Diagnosis, National Cancer Center/National Clinical Research Center for Cancer/Cancer Hospital, Chinese Academy of Medical Sciences and Peking Union Medical College, 17 Panjiayuan South Road, Chaoyang, Beijing 100021, P.R. China

E-mail: maxiaohong@cicams.ac.cn

E-mail: xinmingzh@sina.com

*Contributed equally

Abbreviations: CE-MRI, contrast-enhanced magnetic resonance imaging; MVI, microvascular invasion; HCC, hepatocellular carcinoma; 3D, three-dimensional; AP, arterial phase; PP, portal-venous phase; ROC, receiver operating characteristic; AUC, area under the curve; SM, surgical margin; PET-CT, positron emission tomography-computed tomography; MP, MVI-positive; MN, MVI-negative; AFP, $\alpha$-fetoprotein; LAVA, liver acceleration volume acquisition; MTD, maximum tumor diameter; VOI, volume of interest; GLCM, gray-level co-occurrence matrix; Texscore, texture signature score; ACC, overall accuracy; LRLGLE, LongRunLowGreyLevelEmphasis; RLN, RunLengthNonuniformity; CI, confidence interval

Key words: hepatocellular carcinoma, microvascular invasion, texture analysis, magnetic resonance imaging, prediction group ( $\mathrm{n}=53$ ) and MVI-negative group ( $\mathrm{n}=89$ ). On the basis of three-dimensional texture analysis, 58 features were extracted from the preoperative CE-MR images of arterial-phase (AP) and portal-venous-phase (PP). The t-test or Kruskal-Wallis test, univariate logistic regression analysis and Pearson correlation were applied for feature reduction. Clinical-radiological features were also analyzed. Multivariate logistic regression analysis was used to build the texture model and combined model with clinical-radiological features. The MVI-predictive performance of the models was evaluated using receiver operating characteristic (ROC) analysis and presented using nomogram. Among the clinical features, a significant difference was found in maximum tumor diameter $(\mathrm{P}=0.002)$, tumor differentiation $(\mathrm{P}=0.026)$ and $\alpha$-fetoprotein level $(\mathrm{P}=0.025)$ between the two groups in the training cohort. Four MR texture features in AP and five in PP images were identified through feature reduction. On ROC analysis, the AP texture model showed better diagnostic performance than did the PP model in the validation cohort, with an area under the curve (AUC) of 0.773 vs. 0.623 , sensitivity of 0.750 vs. 0.500 , and specificity of 0.815 vs. 0.926 . Together with the clinical features, the combined model of AP improved the AUC, sensitivity and specificity to $0.810,0.811$ and 0.790 , respectively, which was demonstrated in nomogram. To conclude, model-based texture analysis of CE-MRI could predict MVI in HCC preoperatively and noninvasively, and the AP image shows better predictive efficiency than PP image. The combined model of AP with clinical-radiological features could improve MVI prediction ability.

\section{Introduction}

Hepatocellular carcinoma (HCC) is the sixth most common malignant tumor and the second leading cause of tumor mortality worldwide $(1,2)$, and in 2015 , its incidence and mortality in China ranked the fourth and third, respectively (3). Surgical resection or liver transplantation has been performed for patients with early stage HCC, and is potentially 
curative (4). However, the high postoperative recurrence of HCC remains a common problem (5).

In recent years, many clinical studies have shown that microvascular invasion (MVI) is a significant risk factor for the high rate of recurrence and poor prognosis, and could provide information on which to base clinical treatment (6-8). A previous study indicated that the incidence of MVI ranged from 15.0 to $57.1 \%$ (8). However, no widely recognized definition of MVI is currently available. The commonly accepted histopathological features of MVI include the presence of tumor cells in the portal veins, in large capsular vessels or in vascular spaces lined by endothelial cells $(8,9)$. As demonstrated in a previous study, a wide resection surgical margin (SM) (58\% with $\mathrm{SM} \geq 10 \mathrm{~mm}$ vs. $29 \%$ with $\mathrm{SM}<10 \mathrm{~mm}$ ) may prolong the disease-free survival rate (58 vs. 29\%) (10). Unfortunately, MVI can only be detected after postoperative histopathological examination of the whole surgical specimen, and therefore cannot be used for prediction of treatment benefit. If MVI status could be predicted preoperatively and noninvasively, appropriate treatment could be selected to improve the prognosis.

Recently, a number of studies reported that certain clinical features and morphological characteristics detected on imaging examination could predict the MVI status, including the tumor size, tumor margin, capsule formation and dynamic enhancing pattern (10-12). However, clinical and traditional imaging features were more subjective, lacked quantitative indexes and always showed inter-observer differences, thereby leading to poor reliability of the results that were largely affected by the experience level of the radiologist. Functional imaging modalities, including diffusion-weighted imaging, diffusion kurtosis imaging and positron emission tomography-computed tomography (PET-CT), do not have good predictive power because of their instability and lack of reproducibility $(13,14)$. Thus, the current preoperative prediction of MVI based on imaging signs and/or examinations has limitations, mainly the lack of specificity and practicability. Therefore, developing a method to accurately and quantitatively predict the MVI status of HCC preoperatively is of great clinical significance.

Texture analysis is a widely used image post-processing technique that extracts quantitative features from radiological images to explore the correlation between these features and clinical or histological factors $(15,16)$. Texture analysis enables noninvasive assessment of tumor heterogeneity and provides indirect information on the tumor microenvironment that cannot be obtained with the naked eye (17). More recently, texture analysis has been demonstrated to reveal tumor aggressiveness, thus potentially helping predict the risk of disease progression or recurrence and the response to treatment in many studies; this has also enabled its application in CT, magnetic resonance imaging (MRI) and PET-CT studies (18-21). Several studies have also adopted texture analysis for the differential diagnosis of liver nodules, treatment response evaluation of HCC and prognosis prediction $(22,23)$. In addition, model-based texture analysis has been applied to improve diagnostic efficiency, which could assist clinicians in making treatment decisions (24).

In particular, contrast-enhanced MRI (CE-MRI) is generally used for the diagnosis, treatment evaluation, prognosis estimation, as well as MVI prediction of HCC (12). However, no reports to date have documented the use of texture analysis for MVI prediction of $\mathrm{HCC}$, and none have documented the role of CE-MRI texture analysis in predicting MVI.

The purpose of the present study was to explore the value of CE-MRI texture analysis in preoperatively predicting the MVI status of HCC and determining the diagnostic performance to guide the clinician in choosing appropriate treatment options.

\section{Materials and methods}

Patient selection. The Independent Ethics Committee of the Cancer Hospital, Chinese Academy of Medical Sciences (Beijing, China) approved the current retrospective study and waived the requirement for informed patient consent. Between January 2014 and December 2016, a total of 282 patients with HCC underwent liver MRI examination confirmed by postoperative pathological examinations. The inclusion criteria were as follows: i) Single tumor with a maximum diameter $<5.0 \mathrm{~cm}$, no large vessel invasion and no distant metastasis; ii) underwent radical resection; iii) primary HCC and MVI status confirmed by surgical pathological examination; iv) no other antitumor treatment received before MRI examination and operation; and v) no apparent artifact that may affect imaging analysis. Fig. 1 shows the patient selection flowchart. Consequently, 142 patients were enrolled in the present study and were divided into the MVI-positive (MP) group ( $n=53)$ and the MVI-negative (MN) group $(n=89)$. Next, these 142 patients were divided into two cohorts, including the training cohort with 99 patients who underwent MRI examination between January 2014 and January 2016, and the validation cohort with 43 patients who underwent MRI examination between February 2016 and December 2016. The mean time interval between MRI and surgery was 15 days (range, 7-35 days).

Clinicopathological data. The pathological diagnostic criteria for MVI adopted in this study were reported by Rodríguez-Perálvarez et al (8), namely the presence of tumor emboli in a portal vein, hepatic vein or a large capsule vessel, but not a small bile duct of the surrounded hepatic tissue, as shown in Fig. 2A. Clinical and pathological data collected for analysis included sex, age at diagnosis, $\alpha$-fetoprotein (AFP) level $(\leq 7,7-400$ or $>400 \mathrm{ng} / \mathrm{ml}$ ), tumor location (left, right or caudate lobe), hepatitis B surface antigen (HBsAg) status (positive or negative), hepatitis $\mathrm{C}$ antibody (HCV-Ab) and histologic differentiation (well, moderate or poor). The threshold values chosen for AFP were based on the normal ranges and diagnostic value for HCC used at Cancer Hospital, Chinese Academy of Medical Sciences and Peking Union Medical College.

MR image acquisition. The patients fasted for 6-8 h to empty the gastrointestinal tract before undergoing MRI examination. All of the MR images were acquired using a 3.0-T body MRI system (Discovery MR750 3.0T, GE Medical Systems) equipped with an 8-channel phased-array body coil. The CE-MRI acquisitions were performed with multiphase $3 \mathrm{D}$ spoiled gradient echo liver acceleration volume acquisition (LAVA) sequence, with the following scanning parameters: Repetition time, $2.9 \mathrm{msec}$; echo time, $1.3 \mathrm{msec}$; flip angle, $12^{\circ}$; field of view, 36-42x36-42 cm; matrix, 512x512; section thickness, $4 \mathrm{~mm}$; gap, $0 \mathrm{~mm}$; and number of sections, 36-40. Gadodiamide (Omniscan $0.5 \mathrm{mmol} / \mathrm{ml}$; GE Healthcare) at a standard dose $(0.2 \mathrm{ml} / \mathrm{kg})$ was injected as a 


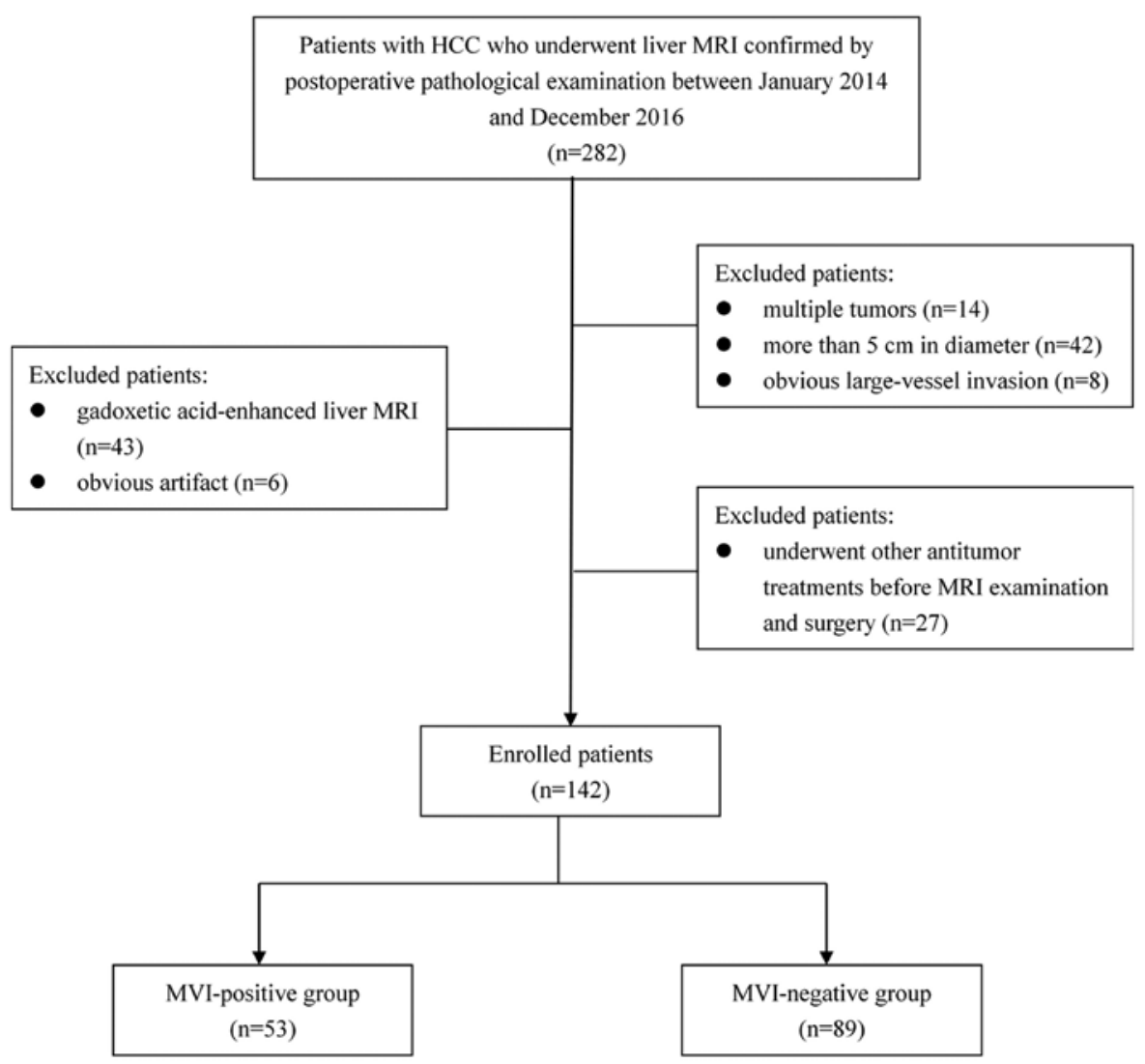

Figure 1. Diagram showing the recruitment of the study population and exclusion criteria. HCC, hepatocellular carcinoma; MRI, magnetic resonance imaging; MVI, microvascular invasion.

bolus through the peripheral veins by using an automatic pump injector at the rate of $3.0 \mathrm{ml} / \mathrm{sec}$, and followed immediately by $20 \mathrm{ml}$ of a $0.9 \%$ sterile saline solution injection. The contrast-enhanced dynamic images were acquired at 15-20 sec (arterial phase, AP), 50-55 sec (portal venous phase, PP) and 85-90 sec (delayed phase) after contrast-agent injection by using the LAVA sequence.

MRI feature analysis. The basic MRI features included the maximum tumor diameter (MTD) (measured by the maximum diameter on the maximum axial section in PP MR image), liver background (cirrhosis or noncirrhosis), tumor encapsulation (peripheral rim of smooth hyperenhancement in PP image), fast wash-in (hyperenhancement of the tumor in the AP), fast wash-out (hypoenhancement of the tumor in the PP) and tumor necrosis (unenhanced areas). The features for each patient were independently evaluated and recorded in a blinded manner by two radiologists with 5 (YJZ) and 3 years (BF) of experience in the interpretation of abdominal MRI to ensure diagnostic accuracy. When a disagreement occurred between the two reviewers during evaluation, a joint review was performed, and consensus data were used for further statistical analysis.

Tumor imaging segmentation and texture analysis. All the MR images were retrieved from the picture archiving and communication system and transferred to a personal computer in the Digital Imaging and Communications in Medicine format. The same two radiologists reviewed and processed the images in a random patient order by using an in-house developed software, Omni-kinetics (version 2.0.10; GE Healthcare
Life Sciences), to obtain texture features. A 3D volume of interest (VOI) of the tumor was manually contoured by the two readers, slightly along the borders of the tumor to include the entire approximated tumor volume.

After generating the VOI, a total of 58 texture features were automatically extracted from the AP and PP images using the Omni-kinetics software. The texture features could be divided into four categories: i) 29 histogram features, ii) 8 gray-level co-occurrence matrix (GLCM) features, iii) 11 Haralick features, and iv) 10 run-length matrix (RLM) features. A detailed list of the features included in the present study is presented in Table I. Fig. 2B shows the diagram of texture analysis.

Statistical analysis and feature reduction. The KolmogorovSmirnov test was used to determine whether the distribution of all the features was normal, and Levene's test was used for identifying the homogeneity of variance. For the clinical-radiological features, a two-tailed unpaired independent t-test was used to compare continuous variables with normal distribution between the MP and MN groups. Categorical variables were compared using the $\chi^{2}$ test or Fisher's exact test.

For texture features, first, an independent t-test or Kruskal-Wallis test was applied one by one. Features with significant differences $(\mathrm{P}<0.05)$ were further analyzed by univariate logistic regression analysis. Features in the univariate logistic regression analysis with $\mathrm{P}<0.05$ were selected. Finally, to eliminate redundant features, Pearson correlation analysis was conducted to remove features with high correlation $(r>0.90)$, which were not considered in the subsequent 
Table I. List of 58 texture analysis parameters.

Texture type

Texture parameters

$\begin{array}{ll}\text { Histogram } & \text { MinIntensity, MaxIntensity, MedianIntensity, MeanValue, stdDeviation, Variance, VolumeCount, } \\ & \text { VoxelValueSum, RMS, Range, MeanDeviation, RelativeDeviation, MinLocation, MaxLocation, } \\ & \text { Skewness, kurtosis, uniformity, Energy, Entropy, FrequencySize, MPP, UPP, Quantile5, Quantile10, } \\ & \text { Quantile25, Quantile50, Quantile75, Quantile90, Quantile95 } \\ & \text { GlcmTotalFrequency, GlcmEnergy, GlcmEntropy, Inertia, Correlation, InverseDifferenceMoment, } \\ \text { GLCM } & \text { ClusterShade, ClusterProminence } \\ \text { Haralick } & \text { HaralickCorrelation, HaraEntropy, AngularSecondMoment, contrast, Hara Variance, sumAverage, } \\ & \text { sumVariance, sumEntropy, differenceVariance, differenceEntropy, inverseDifferenceMoment } \\ \text { RLM } & \text { ShortRunEmphasis, LongRunEmphasis, GreyLevelNonuniformity, RunLengthNonuniformity, } \\ & \text { LowGreyLevelRunEmphasis, HighGreyLevelRunEmphasis, ShortRunLowGreyLevelEmphasis, } \\ & \text { ShortRunHighGreyLevelEmphasis, LongRunLowGreyLevelEmphasis, LongRunHighGreyLevelEmphasis }\end{array}$

RMS, Root mean square; GLCM, gray-level co-occurrence matrix; MPP, mean value of positive pixels; UPP, uniformity of distribution of positive gray-level pixel values; RLM, run-length matrix.
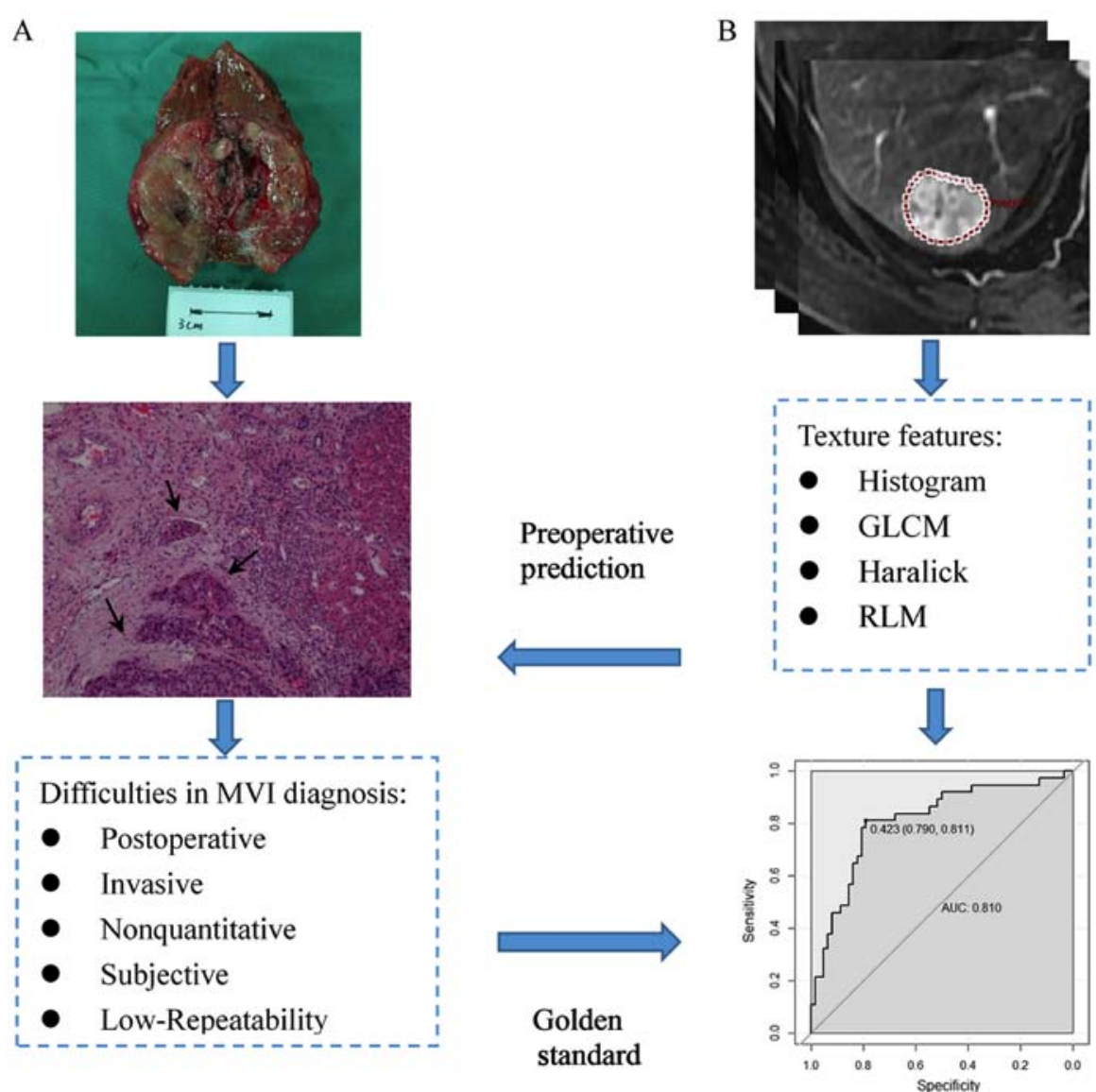

Figure 2. Schematic diagram showing the MVI diagnosis based on pathological examination, prediction using texture analysis and their relationship. (A) Tumor cells could be found in the small vessels near the primary tumor lesion (black arrow). (B) The process of texture analysis includes image segmentation, feature extraction, statistical analysis and model building. MVI, microvascular invasion; GLCM, gray-level co-occurrence matrix; RLM, run-length matrix.

analysis. Features that remained after adjusting for redundancy were entered into model building.

All statistical analyses were performed using $\mathrm{R}$ software (version 3.4.1; R Foundation for Statistical Computing), with a two-tailed probability value. $\mathrm{P}<0.05$ was considered to indicate a statistically significant difference.
Model development. Multivariate logistic regression analysis was applied for model building. First, the texture model was built on the basis of the features selected from a previous step by directly entering. The texture signature score (Texscore), which reflected the overall texture features, was calculated for each patient using the texture model. To involve both 
Table II. The clinical and radiological characteristics of patients in the training and validation cohorts.

A, Clinical characteristics

\begin{tabular}{|c|c|c|c|c|c|c|c|}
\hline \multirow[b]{2}{*}{ Characteristic } & \multicolumn{3}{|c|}{ Training cohort $(n=99)$} & \multicolumn{3}{|c|}{ Validation cohort $(n=43)$} & \multirow[b]{2}{*}{ P-value } \\
\hline & $\operatorname{MP}(n=37)$ & $\mathrm{MN}(\mathrm{n}=62)$ & P-value ${ }^{a}$ & $\operatorname{MP}(n=16)$ & $\mathrm{MN}(\mathrm{n}=27)$ & P-value ${ }^{a}$ & \\
\hline Sex & & & $0.931^{\mathrm{c}}$ & & & $0.723^{\mathrm{c}}$ & $0.805^{\mathrm{c}}$ \\
\hline Male & 32 & 54 & & 15 & 23 & & \\
\hline Female & 5 & 8 & & 1 & 4 & & \\
\hline Mean age $\pm S D$, years & $57.49 \pm 9.56$ & $56.45 \pm 9.71$ & $0.607^{\mathrm{e}}$ & $55.88 \pm 8.63$ & $55.41 \pm 8.00$ & $0.858^{\mathrm{e}}$ & $0.456^{\mathrm{e}}$ \\
\hline $\mathrm{AFP}, \mathrm{ng} / \mathrm{ml}$ & & & $0.025^{\mathrm{c}}$ & & & $0.038^{\mathrm{c}}$ & $0.715^{\mathrm{c}}$ \\
\hline$\leq 7$ & 9 & 32 & & 5 & 16 & & \\
\hline $7-400$ & 17 & 20 & & 5 & 9 & & \\
\hline$>400$ & 11 & 10 & & 6 & 2 & & \\
\hline Location & & & $0.561^{\mathrm{c}}$ & & & $0.372^{\mathrm{c}}$ & $0.581^{\mathrm{c}}$ \\
\hline Right & 31 & 49 & & 10 & 23 & & \\
\hline Left & 6 & 13 & & 6 & 4 & & \\
\hline HBsAg & & & $0.325^{\mathrm{c}}$ & & & $0.614^{\mathrm{c}}$ & $0.668^{\mathrm{c}}$ \\
\hline Positive & 26 & 49 & & 12 & 22 & & \\
\hline Negative & 11 & 13 & & 4 & 5 & & \\
\hline $\mathrm{HCV}-\mathrm{Ab}$ & & & $1.000^{\mathrm{c}}$ & & & $1.000^{\mathrm{d}}$ & $0.989^{c}$ \\
\hline Positive & 1 & 3 & & 0 & 1 & & \\
\hline Negative & 36 & 59 & & 16 & 26 & & \\
\hline Differentiation & & & $0.026^{\mathrm{c}}$ & & & $0.034^{\mathrm{d}}$ & $0.565^{\mathrm{c}}$ \\
\hline Well & 2 & 3 & & 0 & 4 & & \\
\hline Moderate & 19 & 47 & & 9 & 20 & & \\
\hline Poor & 16 & 12 & & 7 & 3 & & \\
\hline
\end{tabular}

B, Radiological characteristics

\begin{tabular}{|c|c|c|c|c|c|c|c|}
\hline \multirow[b]{2}{*}{ Characteristic } & \multicolumn{3}{|c|}{ Training cohort $(\mathrm{n}=99)$} & \multicolumn{3}{|c|}{ Validation cohort $(n=43)$} & \multirow[b]{2}{*}{ P-value } \\
\hline & $\operatorname{MP}(n=37)$ & $\mathrm{MN}(\mathrm{n}=62)$ & P-value & $\operatorname{MP}(n=16)$ & $\mathrm{MN}(\mathrm{n}=27)$ & P-value & \\
\hline $\mathrm{MTD}, \mathrm{cm}$ & $3.82 \pm 0.88$ & $3.21 \pm 0.94$ & $0.002^{\mathrm{e}}$ & $3.75 \pm 0.80$ & $2.92 \pm 0.62$ & $<0.001^{\mathrm{e}}$ & $0.231^{\mathrm{e}}$ \\
\hline Background liver & & & $0.690^{c}$ & & & $0.358^{\mathrm{c}}$ & $0.553^{c}$ \\
\hline Noncirrhosis & 14 & 21 & & 3 & 10 & & \\
\hline Cirrhosis & 23 & 41 & & 13 & 17 & & \\
\hline Tumor encapsulation & & & $0.805^{\mathrm{c}}$ & & & $0.362^{c}$ & $0.534^{\mathrm{c}}$ \\
\hline Absent & 11 & 17 & & 2 & 8 & & \\
\hline Present & 26 & 45 & & 14 & 19 & & \\
\hline Fast wash-in & & & $0.292^{c}$ & & & $0.929^{c}$ & $0.742^{\mathrm{c}}$ \\
\hline Yes & 30 & 55 & & 14 & 22 & & \\
\hline No & 7 & 7 & & 2 & 5 & & \\
\hline Fast wash-out & & & $0.409^{c}$ & & & $0.534^{\mathrm{c}}$ & $0.720^{c}$ \\
\hline Yes & 24 & 35 & & 11 & 16 & & \\
\hline No & 13 & 27 & & 5 & 11 & & \\
\hline Tumor necrosis & & & $0.638^{c}$ & & & $0.372^{c}$ & $0.668^{c}$ \\
\hline Absent & 29 & 46 & & 11 & 23 & & \\
\hline Present & 8 & 16 & & 5 & 4 & & \\
\hline
\end{tabular}

MP, microvascular invasion-positive; MN, microvascular invasion-negative; AFP, $\alpha$-fetoprotein; HBsAg, hepatitis B surface antigen; MTD, maximum tumor diameter; HCV-Ab, hepatitis $\mathrm{C}$ antibody. ${ }^{\mathrm{a}} \mathrm{MP}$ vs. MN; ${ }^{\mathrm{b}}$ Training cohort vs. validation cohort; ${ }^{\mathrm{C}} \mathrm{P}-\mathrm{values}$ calculated using the $\chi^{2}$ test; ${ }^{\mathrm{d}} \mathrm{P}$-values calculated using Fisher's exact test; ${ }^{\mathrm{e}} \mathrm{P}$-values calculated using the independent t-test. 
Table III. Univariate logistic regression analysis of the clinical and texture features to predict the microvascular invasion status in the training cohort.

\begin{tabular}{|c|c|c|c|c|}
\hline \multirow[b]{3}{*}{ Feature } & \multicolumn{3}{|c|}{ OR } & \multirow[b]{3}{*}{ P-value } \\
\hline & \multirow[b]{2}{*}{ Value } & \multicolumn{2}{|c|}{$95 \% \mathrm{CI}$} & \\
\hline & & Lower & Upper & \\
\hline \multicolumn{5}{|l|}{ AP texture features } \\
\hline Uniformity & $1.209 \times 10^{4}$ & 6.602 & $4.605 \times 10^{7}$ & 0.019 \\
\hline Energy & $3.110 \times 10^{121}$ & $4.556 \times 10^{19}$ & $1.820 \times 10^{229}$ & 0.022 \\
\hline Entropy & $1.123 \times 10^{-1}$ & $1.991 \times 10^{-2}$ & $5.647 \times 10^{-1}$ & 0.010 \\
\hline UPP & $3.110 \times 10^{121}$ & $4.556 \times 10^{19}$ & $1.820 \times 10^{229}$ & 0.022 \\
\hline ClusterShade & 1.000 & 1.000 & 1.000 & 0.046 \\
\hline ClusterProminence & 1.000 & 1.000 & 1.000 & 0.005 \\
\hline GreyLevelNonuniformity & 1.003 & 1.001 & 1.007 & 0.023 \\
\hline LowGreyLevelRunEmphasis & 0.000 & 0.000 & $3.320 \times 10^{-192}$ & 0.034 \\
\hline ShortRunLowGreyLevelEmphasis & 0.000 & 0.000 & $2.140 \times 10^{-255}$ & 0.035 \\
\hline LongRunLowGreyLevelEmphasis & 0.000 & 0.000 & $2.060 \times 10^{-79}$ & 0.036 \\
\hline \multicolumn{5}{|l|}{ PP texture features } \\
\hline MinIntensity & $9.973 \times 10^{-1}$ & $9.947 \times 10^{-1}$ & $9.995 \times 10^{-1}$ & 0.025 \\
\hline VolumeCount & 1.000 & 1.000 & 1.000 & 0.027 \\
\hline Uniformity & $1.087 \times 10^{-3}$ & $9.070 \times 10^{-7}$ & $6.241 \times 10^{-1}$ & 0.044 \\
\hline FrequencySize & 1.000 & 1.000 & 1.000 & 0.027 \\
\hline GlcmTotalFrequency & 1.000 & 1.000 & 1.000 & 0.026 \\
\hline GlcmEntropy & $6.627 \times 10^{-1}$ & $4.454 \times 10^{-1}$ & $9.462 \times 10^{-1}$ & 0.030 \\
\hline HaraEntroy & $3.266 \times 10^{5}$ & $2.273 \times 10^{1}$ & $1.286 \times 10^{10}$ & 0.013 \\
\hline AngularSecondMoment & 0.000 & 0.000 & $4.329 \times 10^{-94}$ & 0.026 \\
\hline sumAverage & $1.301 \times 10^{-2}$ & $2.238 \times 10^{-4}$ & $5.748 \times 10^{-1}$ & 0.029 \\
\hline sumEntropy & $1.896 \times 10^{4}$ & 4.127 & $1.996 \times 10^{8}$ & 0.028 \\
\hline GreyLevelNonuniformity & 1.003 & 1.001 & 1.006 & 0.024 \\
\hline RunLengthNonuniformity & 1.000 & 1.000 & 1.000 & 0.025 \\
\hline
\end{tabular}

OR, odds ratio; CI, confidence interval; UPP, uniformity of distribution of positive gray-level pixel values; AP, arterial phase; PP, portal venous phase.

texture features and clinical-radiological features to improve performance, the combined model was built on the basis of the Texscore as well as other significant clinical-radiological features in both AP and PP.

Receiver operating characteristic (ROC) analysis and the nomogram plot of the model. The performance of the texture and combined models was analyzed both in the training and validation cohorts by using a ROC curve quantified by the area under the curve (AUC), sensitivity, specificity and overall accuracy (ACC). The cutoff point was calculated at the maximized value of the Youden index (sensitivity + specificity-1) (25). Nomogram of the combined model was formulated in order to visualize model efficiency.

\section{Results}

Clinical and radiological features. Of the 142 patients enrolled in the study, 124 patients were male and 18 were female. Median age was 57 years (range, 34-80 years). The results revealed that MTD $(\mathrm{P}=0.002)$, serum AFP level $(\mathrm{P}=0.025)$ and tumor differentiation $(\mathrm{P}=0.026)$ showed significant differences between the MP and MN groups in the training cohort. The MP group had greater MTD than did the MN group $(3.82 \pm 0.88$ vs. $3.21 \pm 0.94 \mathrm{~cm})$, and also tended to have a higher serum AFP level and lower tumor differentiation. This result was also confirmed in the validation cohort. The characteristics of the training and validation cohorts showed no significant differences (all $\mathrm{P}>0.05$ ). The detailed clinical and radiological features of the patients in the training and validation cohorts are listed in Table II.

Texture feature reduction. First, the Kruskal-Wallis test revealed that 19 texture features in AP and 18 in PP showed significant differences $($ all $\mathrm{P}<0.05)$ between the MP and $\mathrm{MN}$ groups. In the univariate logistic regression analysis, 10 texture features in AP and 12 in PP showed a potential predictive value $(\mathrm{P}<0.05)$ to discriminate between the MP and MN groups. Detailed results of the univariate logistic regression analysis are demonstrated in Table III. Finally, a Pearson 

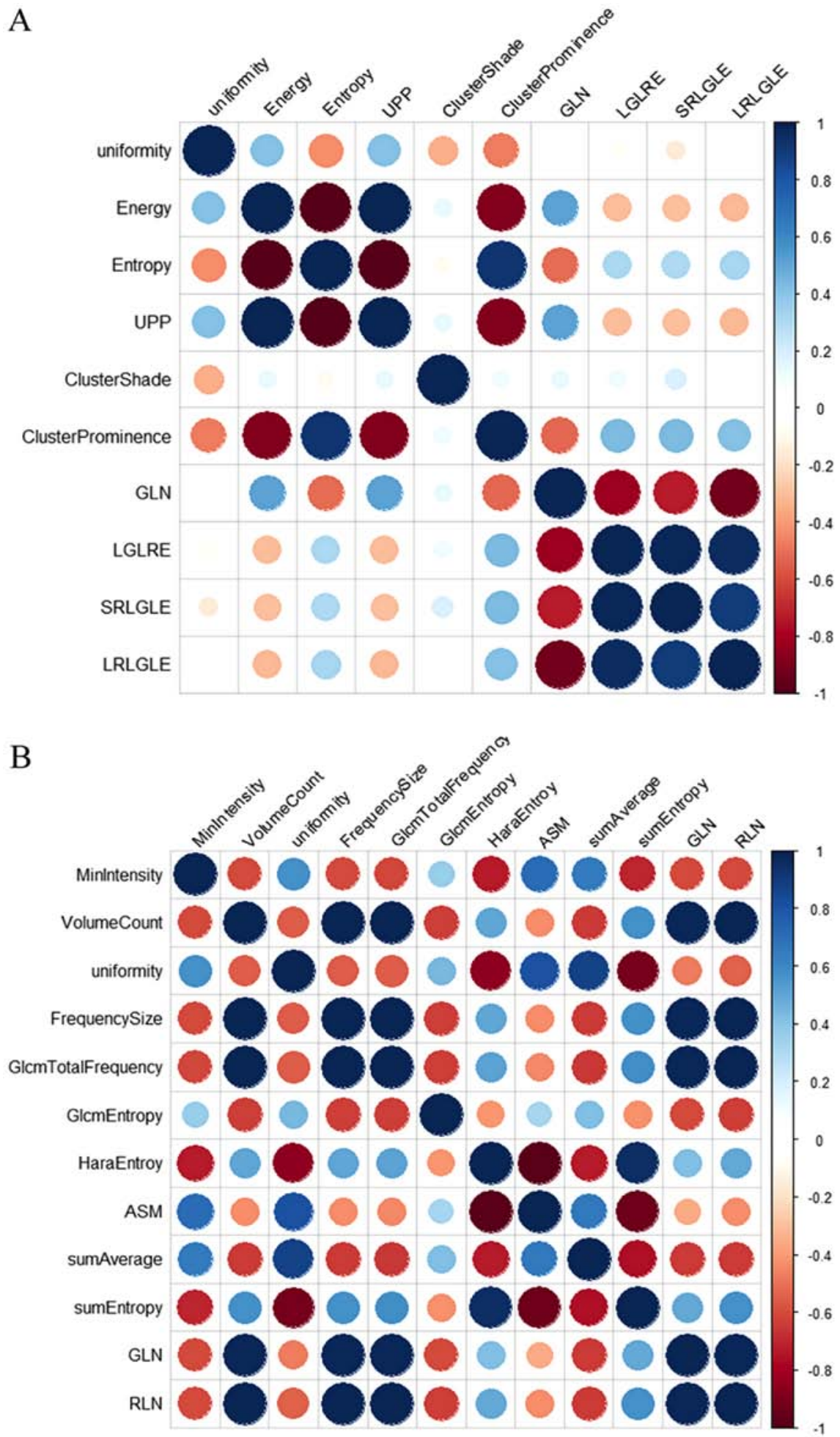

Figure 3. Pearson correlation matrix plot for (A) 10 texture features in the arterial phase and (B) 12 texture features in the portal venous phase of the training cohort. Blue circles indicate positive correlation, red circles negative correlation. The larger the circle and the darker the color, the higher is the correlation between two texture features. GLN, GreyLevelNonuniformity; LRLGLE, LongRunLowGreyLevelEmphasis; LGLRE, LowGreyLevelRunEmphasis; SRLGLE, ShortRunLowGreyLevelEmphasis; RLN, RunLengthNonuniformity; ASM, AngularSecondMoment; UPP, uniformity of distribution of positive gray-level pixel values.

correlation matrix was then calculated from the above features to detect highly collinear texture features (Fig. 3). After eliminating significant highly correlated features (defined as Pearson's $r \geq 0.90$ ), four texture features in AP, including uniformity, ClusterProminence, ClusterShade and LongRun LowGreyLevelEmphasis (LRLGLE), and five in PP, including
MinIntensity, GlcmEntropy, sumAverage, sumEntropy and RunLengthNonuniformity (RLN), were retained for the subsequent analysis.

Multivariate logistic regression analysis and texture model building. Texture features identified from the above analysis 
in AP and PP were entered into the multivariate logistic regression model to build the texture predictive model. The detailed results of the multivariate logistic regression analysis are shown in Table IV.

The texture signature score (Texscore) of each patient in AP and PP could be calculated using the formula based on multivariate logistic regression model as follows: Texscore $(\mathrm{AP})=0.455+1.30 \mathrm{x}$ uniformity $+0.524 \mathrm{x}$ ClusterP rominence $+0.593 \times$ ClusterShade $+0.494 \times$ LRLGLE; and Texscore $(\mathrm{PP})=7.310+0.732 \times$ GlcmEntropy $+0.002 \times$ sumEntropy $+2.230 \times 10^{5} \times$ sumAverage $+0.999 \times$ MinIntensity $+1.000 \times$ RLN.

Development of the combined model. In the training cohort, two combined models were built to predict the MVI status by clinical-radiological features and the Texscore generated above in AP and PP separately (Table V). In the combined model in AP, only the texture signature showed significant [odds ratio (OR), 2.552; $\mathrm{P}=0.003$ ], but none of the clinical-radiological features showed significance. In the combined model in PP, MTD (OR, 0.759; $\mathrm{P}=0.032)$ and AFP (OR, 1.307; $\mathrm{P}=0.027$ and $\mathrm{OR}, 1.768 ; \mathrm{P}=0.017)$ showed significance, but the texture signature and differentiation did not show significance.

Nomograms in AP and PP. The nomograms integrating the features included in the multivariate logistic regression analysis are displayed in Fig. 4, which could visualize the weight of different features in combined model. The concordance indexes of the nomograms for MVI predictions in AP and PP were 0.810 [95\% confidence interval (CI), 0.718-0.902] and 0.799 (95\% CI, 0.710-0.889), respectively.

Predictive performance of MVI. For the training cohort, the ROC curves illustrating the predictive performance of the texture and combined models in predicting the MVI status are provided in Fig. 5. The AUC of the texture model was 0.765 vs. 0.707 in AP and PP, respectively. The AUC of the combined model was 0.810 vs. 0.799 in AP and PP, respectively. The AUC, ACC, sensitivity and specificity for each model are summarized in Table VI.

The validation cohort was used to verify the accuracy of the model built in the training cohort. The ROC curves of the texture and combined models for predicting the MVI status are shown in Fig. 6. The AUC of the texture model was 0.773 vs. 0.623 in AP and PP, respectively. The AUC of the combined model was 0.794 vs. 0.706 in AP and PP, respectively. The AUC, ACC, sensitivity and specificity for each model are summarized in Table VI.

\section{Discussion}

In the present study, a combined model was developed based on preoperative 3D CE-MRI texture and clinical-radiological features to predict MVI with a satisfactory discriminatory performance. The results indicated that texture analysis is a potentially useful adjunct for predicting MVI, and adding clinical-radiological data could slightly improve the predictive ability. In addition, the AP texture features performed better than the PP texture features in MVI prediction.
Table IV. Multivariate logistic regression analysis of the texture parameters in predicting the microvascular invasion status in the arterial and portal venous phases in the training cohort.

\begin{tabular}{lcc} 
A, Arterial phase & & \\
\hline Feature & OR & P-value \\
\hline (Intercept) $^{\mathrm{a}}$ & 0.455 & 0.003 \\
Uniformity $_{\text {ClusterShade }}$ & 1.301 & 0.387 \\
ClusterProminence & 0.524 & 0.059 \\
LRLGLE & 0.593 & 0.262 \\
& 0.494 & 0.170 \\
\hline
\end{tabular}

$\mathrm{B}$, Portal venous phase

\begin{tabular}{lcc}
\hline Feature & OR & P-value \\
\hline (Intercept) $^{\mathrm{a}}$ & 7.310 & 0.754 \\
GlcmEntropy $_{\text {sumEntropy }}$ & 0.732 & 0.146 \\
sumAverage & 0.002 & 0.474 \\
MinIntensity & $2.230 \mathrm{e}+05$ & 0.124 \\
RLN & 0.999 & 0.563 \\
\end{tabular}

antercept is the constant term in the logistic regression equation. OR, odds ratio; LRLGLE, LongRunLowGreyLevelEmphasis; RLN, RunLengthNonuniformity.

Table V. Multivariate logistic regression analysis of the combined clinical-radiological and texture features to predict the microvascular invasion status in the AP and PP in the training cohort.

\begin{tabular}{lccccc}
\hline & \multicolumn{2}{c}{ AP Model } & & \multicolumn{2}{c}{ PP Model } \\
\cline { 2 - 3 } \cline { 6 - 6 } Features & OR & P-value & & OR & P-value \\
\hline Intercept) & 0.257 & 0.282 & & -3.068 & 0.052 \\
MTD, cm & 1.395 & 0.242 & & 0.759 & 0.032 \\
Differentiation & & & & & \\
$\quad$ Moderate vs. poor & 0.559 & 0.284 & & -0.838 & 0.179 \\
Well vs. low & 0.672 & 0.747 & & -0.697 & 0.560 \\
a-fetoprotein, ng/ml & & & & \\
7-400 vs. $\leq 7$ & 1.801 & 0.298 & & 1.307 & 0.027 \\
$>400$ vs. $\leq 7$ & 3.771 & 0.053 & & 1.768 & 0.017 \\
Texscore & 2.552 & 0.003 & & 0.449 & 0.294
\end{tabular}

${ }^{a}$ Intercept is the constant term in the logistic regression equation, and is not a clinical-radiological feature. AP, arterial phase; PP, portal venous phase; OR, odds ratio; MTD, maximum tumor diameter.

The present study involved a total of 142 patients, 53 of which were MVI-positive patients and 89 MVI-negative. The MVI-positive rate was consistent with the reported rate in 
Table VI. Predictive performance of the texture and combined models in predicting the microvascular invasion status in the training and validation cohorts.

\begin{tabular}{|c|c|c|c|c|c|c|c|c|c|}
\hline \multirow[b]{2}{*}{ Model } & \multirow[b]{2}{*}{ Phase } & \multicolumn{4}{|c|}{ Training cohort $(\mathrm{n}=99)$} & \multicolumn{4}{|c|}{ Validation cohort $(n=43)$} \\
\hline & & AUC & $\mathrm{ACC}$ & SEN & SPE & AUC & $\mathrm{ACC}$ & SEN & SPE \\
\hline \multirow[t]{2}{*}{ Texture } & $\mathrm{AP}$ & 0.765 & 0.768 & 0.730 & 0.790 & 0.773 & 0.791 & 0.750 & 0.815 \\
\hline & PP & 0.707 & 0.727 & 0.622 & 0.790 & 0.623 & 0.767 & 0.500 & 0.926 \\
\hline \multirow[t]{2}{*}{ Combined } & AP & 0.810 & 0.798 & 0.811 & 0.790 & 0.794 & 0.837 & 0.812 & 0.852 \\
\hline & PP & 0.799 & 0.758 & 0.730 & 0.774 & 0.706 & 0.721 & 0.750 & 0.704 \\
\hline
\end{tabular}

AP, arterial phase; PP, portal venous phase; AUC, area under the curve; ACC, overall accuracy; SEN, sensitivity; SPE, specificity.

A

Points

Texscore

MTD

Differentiation

AFP

Total points

Probability

B

Points

Texscore

MTD

Differentiation

AFP

Total points

Probability

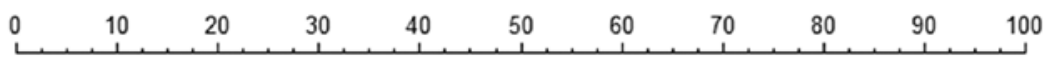

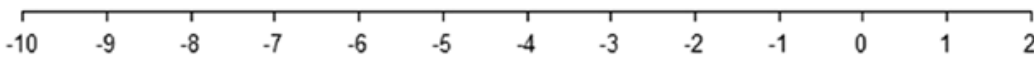

तापामाप

$\begin{array}{llll}1 & 2.5 & 4 & 5.5\end{array}$


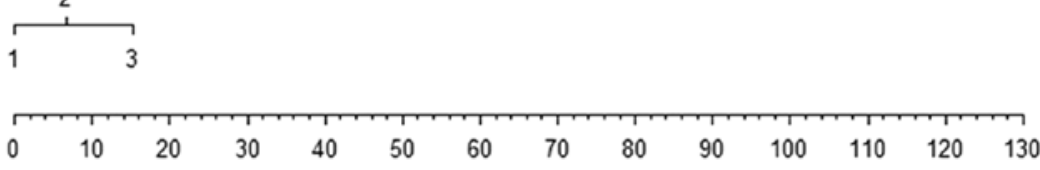

$\begin{array}{llllll}0.1 & 0.3 & 0.5 & 0.7 & 0.9 & 0.95\end{array}$

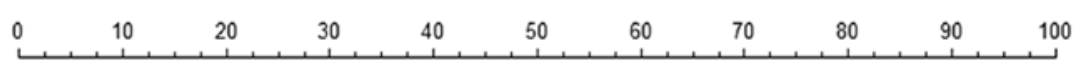

\begin{tabular}{|c|c|c|c|c|c|c|}
\hline-2.5 & -2 & & -1 & -0.5 & 0 & 0. \\
\hline
\end{tabular}

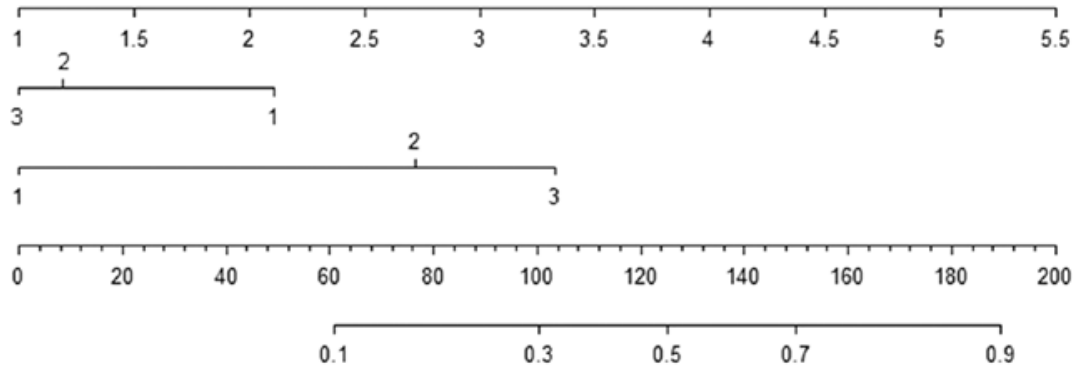

Figure 4. Nomograms for predicting the microvascular invasion status of hepatocellular carcinoma in (A) the arterial phase and (B) portal venous phase using the texture signature and clinical-radiological features. AFP) levels 1,2 and 3 stand for $\leq 7,7-400$ and $>400 \mathrm{ng} / \mathrm{ml}$, respectively. Differentiation levels 1,2 and 3 stand for poor, moderate and well differentiation, respectively. MTD, maximum tumor diameter; AFP, $\alpha$-fetoprotein.

the literature (8), which was approximately $30.0 \%$. Previous studies have reported that the tumor size of HCC was one of the most important predictive factors for MVI $(26,27)$. This is consistent with the results of the current study. In the training cohort, MTD in the MP group $(3.82 \pm 0.88 \mathrm{~cm})$ was significantly greater than that in the MN group $(3.21 \pm 0.94 \mathrm{~cm})$ $(\mathrm{P}=0.002)$, since tumor size is an important feature of tumor burden. Moreover, patients with poorer tumor histological differentiation were observed in the MP group $(\mathrm{P}=0.026)$, and this feature has been reported to be a predictor of MVI in previous studies $(28,29)$, possibly due to the high invasiveness of poorly differentiated tumors. In the present study, the result showed that the MP group tended to have higher AFP levels $(\mathrm{P}=0.025)$. Serum AFP level is considered a marker of HCC, which has also been reported to correlate with MVI (30). Nevertheless, the mechanism needs to be further 
A

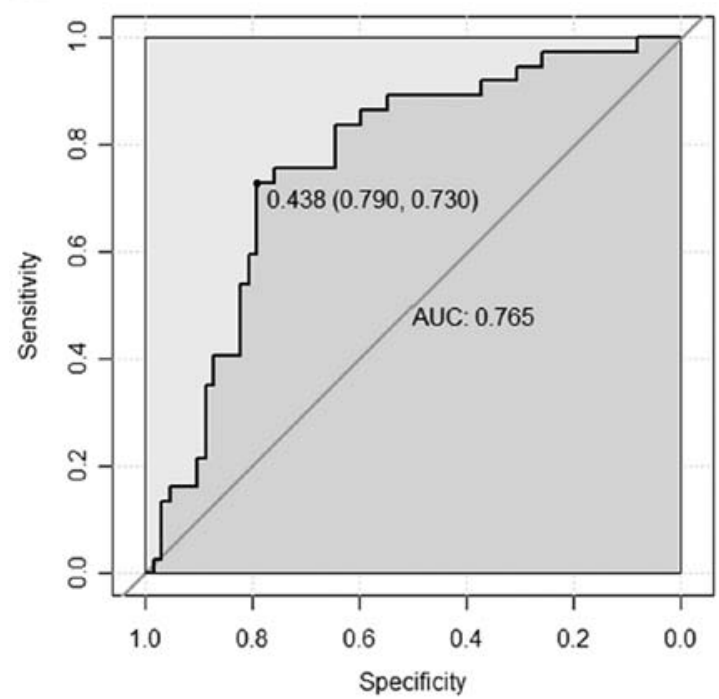

C Combined model of the training cohort in AP

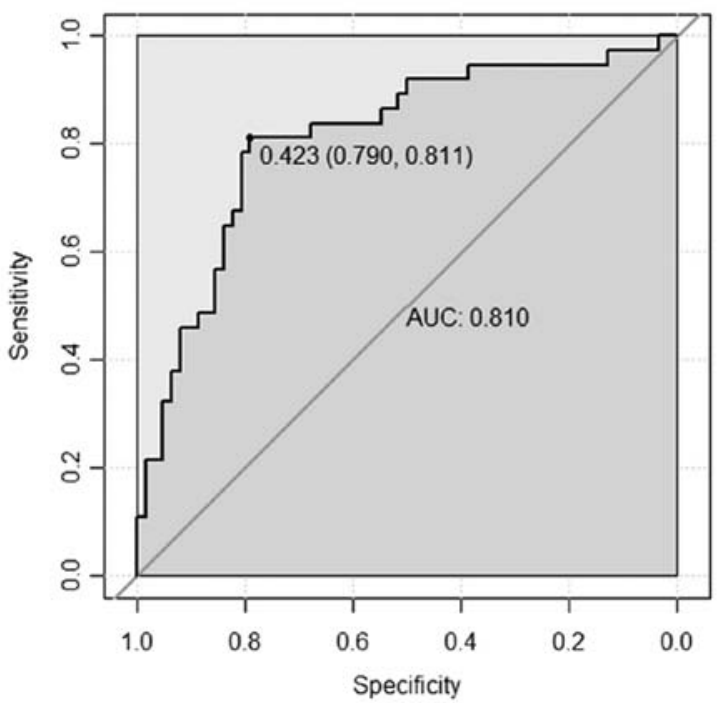

B Texture model of the training cohort in PP

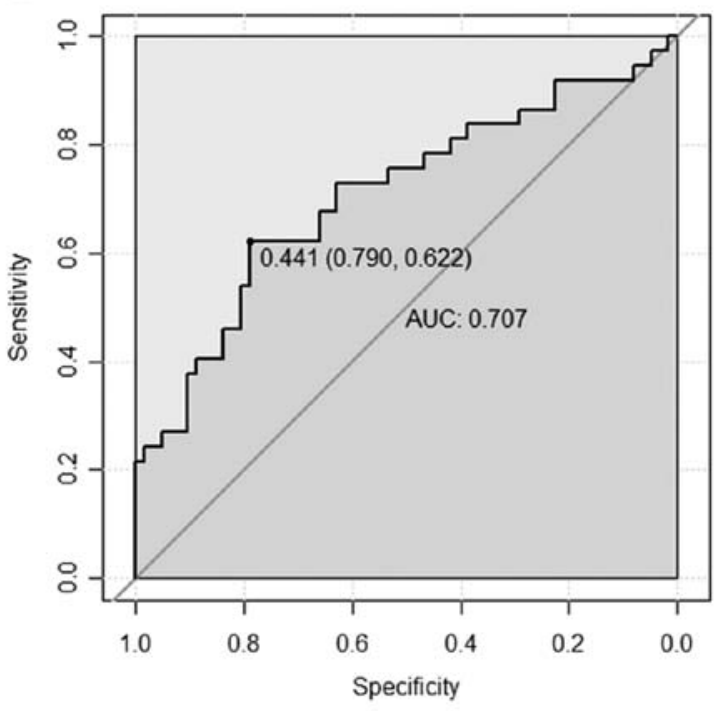

D Combined model of the training cohort in PP

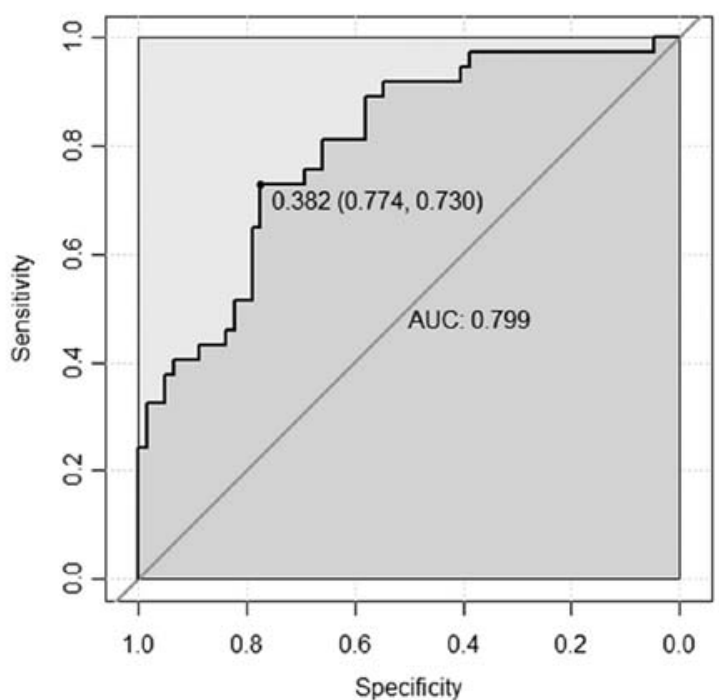

Figure 5. ROC curves for the texture and combined models in predicting the microvascular invasion status in the training cohort. The solid dots represent the optimal cutoff values (specificity, sensitivity) for discrimination. The AUC for the texture models in (A) AP and (B) PP are 0.765 and 0.707 , respectively. The AUC for the combined models in (C) AP and (D) PP are 0.810 and 0.799, respectively. ROC, receiver operating characteristic; AUC, area under the curve; AP, arterial phase; PP, portal-venous phase.

clarified. In the validation cohort, the above results were confirmed again.

CE-MRI texture analysis of the AP and PP images was used to build models for predicting the MVI status, and the AP images showed better predictive ability than did the PP images. Four texture features in AP (uniformity, ClusterProminence, ClusterShade and LRLGLE) and five in PP (MinIntensity, GlcmEntropy, sumAverage, sumEntropy and RLN) were entered into the multivariate logistic regression analysis. These features frequently appeared in texture or radiomics research and showed noteworthy diagnostic and predictive efficiency, which could be explained by their definitions $(31,32)$. All these features reflect the heterogeneity in an image from a different aspect, and were thus related to tumor heterogeneity from a clinical point of view. Previous studies have shown that tumor heterogeneity is related to tumor differentiation, angiogenesis and prognosis $(33,34)$. On the basis of the current results, tumor heterogeneity may be associated with the occurrence of MVI.

In the multivariate logistic regression analysis, the directly enter mode was used to build the predictive model, but the $\mathrm{P}$-values of the texture features in the texture model were not significant. The possible explanation was that all parameters were equally important, and hence, no significant texture features were observed in the regression. The combined model was also built by adding the clinical-radiological features to the multivariate logistic regression. The nomogram was used to visualize the combined model and to reveal the weight of the clinical-radiological and texture features. For clinical use of the model, the total scores of each patient could be calculated based on the nomogram. High scores corresponded to a high probability of MVI occurrence. The nomogram showed that 
A Texture model of the validation cohort in AP

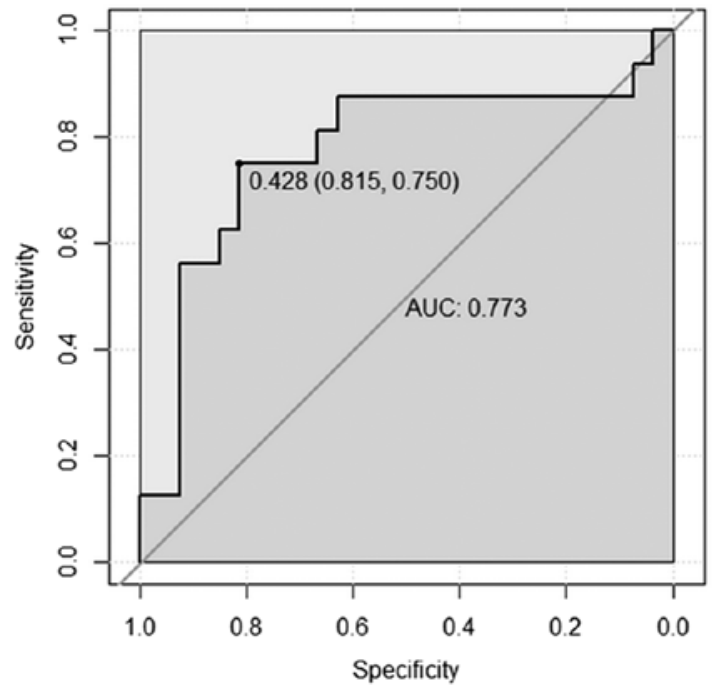

C Combined model of the validation cohort in AP

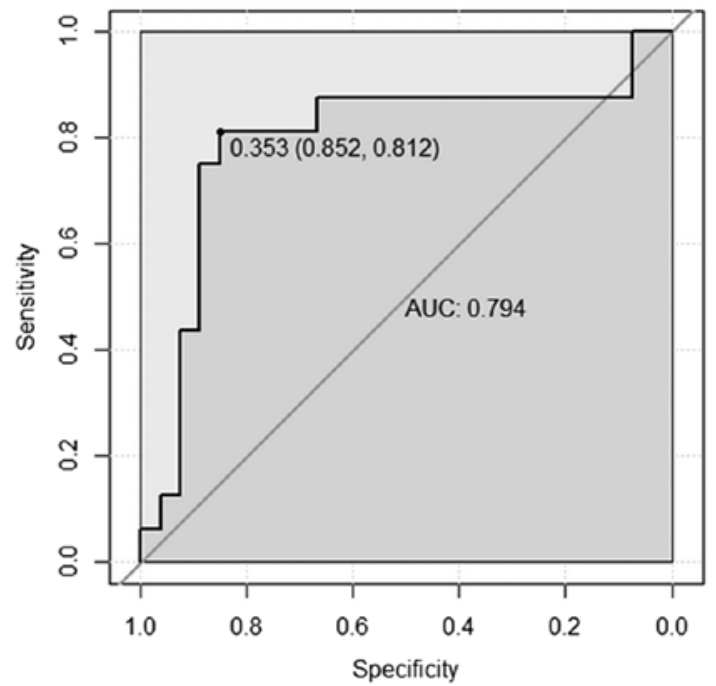

B Texture model of the validation cohort in PP

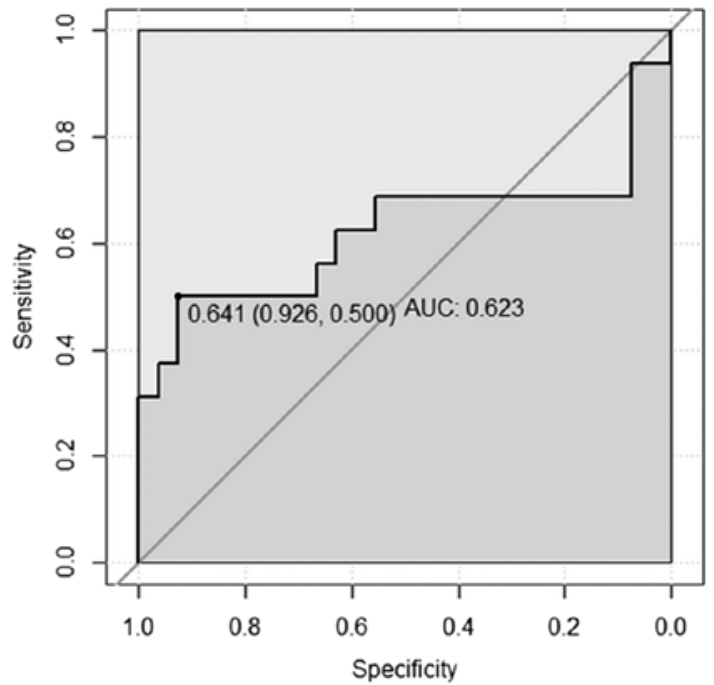

D Combined model of the validation cohort in PP

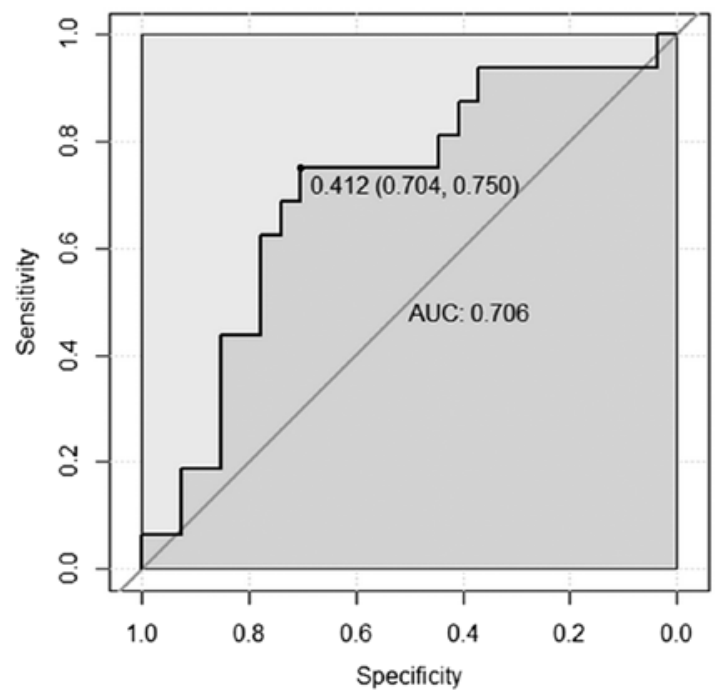

Figure 6. ROC curves for the texture and combined models in predicting the microvascular invasion status in the validation cohort. The solid dots represent the optimal cutoff values (specificity, sensitivity) for discrimination. The AUC for the texture models in the (A) AP and (B) PP are 0.773 and 0.623 , respectively. The AUC for the combined models in (C) AP and (D) PP are 0.794 and 0.706, respectively. ROC, receiver operating characteristic; AUC, area under the curve; AP, arterial phase; PP, portal-venous phase.

the texture signature accounted for a higher proportion in the total points than did the clinical-radiological features in AP.

In the ROC analysis, the combined model showed a little better predictive performance than did the texture model in the validation cohort both in AP (AUC, 0.794 vs. 0.773 ; specificity, 0.852 vs. 0.815 ; sensitivity, 0.812 vs. 0.750 ) and in PP (AUC, 0.706 vs. 0.623; specificity, 0.704 vs. 0.926; sensitivity, 0.750 vs. 0.500 ). The predictive ability was better in AP than in PP. The possible explanations might be that the blood supply to the HCC is mainly dependent on the hepatic artery, and that the AP image could more clearly reflect the changes in small blood vessels in the HCC. Moreover, when MVI occurs, the local hemodynamics of the liver tissue around the tumor changes. Therefore, the changes in MRI texture features in AP were more obvious than those in PP.

From a clinical perspective, the results of the present study suggest that CE-MRI texture analysis may be an option for preoperatively predicting MVI in HCC. This could alert pathologists to conduct more detailed pathological examinations, particularly when preoperative texture analysis suggests a high possibility of MVI occurrence. Meanwhile, predicting the possibility of MVI occurrence could also help clinicians select more suitable surgical procedures for HCC patients. A number of studies have shown that anatomic resection $(35,36)$ and wide resection SM (10) may provide radical treatment in HCC patients with MVI. Intrahepatic metastases resulting from MVI could also be reduced, thereby improving the prognosis.

The present study had the following advantages. First, to our knowledge, this was the first study to use 3D MRI texture features to build a model for predicting the MVI status preoperatively and noninvasively. The 3D VOI may have a better predictive performance since MVI could occur in every slice, and $3 \mathrm{D}$ feature analysis considers all of the available slices 
with abundant information. Previous studies have also shown that 3D features improved the diagnostic accuracy than did two-dimensional features $(37,38)$. Second, both the AP and PP images were analyzed to compare their predictive performance. Third, training and validation cohorts were used, making the model more objective. Therefore, the process and method established in the present study provide a basis for further computer-aided and artificial-intelligence analyses for predicting MVI in HCC.

The current study also had several limitations. First, this was a retrospective study with a single-center design; therefore, selection bias was unavoidable. Second, the sample size was small for texture analysis. More cases are needed to verify the results. Third, the manual segmentation of tumors may have introduced a certain amount of subjectivity. The MVI grade in the positive group was not taken into account. Forth, the present study did not include des- $\gamma$-carboxy-prothrombin (DCP) as it was not tested routinely in our hospital, although a study has reported that DCP was useful for prediction of MVI (39). At last, no follow-up and survival analysis were performed, and hence, the relationship between prognosis and texture features could not be further clarified.

In conclusion, model-based texture analysis of CE-MRI could predict MVI in HCC preoperatively and noninvasively. The AP image shows better predictive efficiency than PP image. The combined model of AP with clinical-radiological features could improve MVI prediction ability. It may be of value to clinicians in objectively selecting appropriate treatment strategies and as an individualized predictive tool for improving clinical outcomes.

\section{Acknowledgements}

Not applicable.

\section{Funding}

This study was supported by the Peking Union Medical College Youth Fund and the Fundamental Research Funds for the Central Universities (grant no. 2017320010), the Chinese Academy of Medical Sciences (CAMS) Research Fund (grant no. ZZ2016B01) and the CAMS Innovation Fund for Medical Sciences (grant no. 2016-I2M-1-001).

\section{Availability of data and materials}

The datasets used and/or analyzed during the current study are available from the corresponding author on reasonable request.

\section{Authors' contributions}

YJZ, SW, LMW, XHM and XMZ designed this study. YJZ, BF and LMW acquired the patients and searched the database. YJZ and BF participated in the process of texture analysis, including segmentation and features extraction. JFW contributed to the analysis and interpretation of data, including statistical analysis, biostatistics and computational analysis. YJZ and XHM were major contributors in writing the manuscript. All authors read and approved the final manuscript.

\section{Ethics approval and consent to participate}

The independent ethics committee of the Cancer Hospital, Chinese Academy of Medical Sciences approved this retrospective study and waived the requirement for informed patient consent.

\section{Patient consent for publication}

Not applicable.

\section{Competing interests}

The authors declare that they have no competing interests.

\section{References}

1. McGlynn KA, Petrick JL and London WT: Global epidemiology of hepatocellular carcinoma: An emphasis on demographic and regional variability. Clin Liver Dis 19: 223-238, 2015.

2. Torre LA, Bray F, Siegel RL, Ferlay J, Lortet-Tieulent J and Jemal A: Global cancer statistics, 2012. CA Cancer J Clin 65: 87-108, 2015.

3. Chen W, Zheng R, Baade PD, Zhang S, Zeng H, Bray F, Jemal A, Yu XQ and He J: Cancer statistics in China, 2015. CA Cancer J Clin 66: 115-132, 2016.

4. Dhir M, Melin AA, Douaiher J, Lin C, Zhen WK, Hussain SM, Geschwind JF, Doyle MB, Abou-Alfa GK and Are C: A review and update of treatment options and controversies in the management of hepatocellular carcinoma. Ann Surg 263: 1112-1125, 2016.

5. Forner A, Llovet JM and Bruix J: Hepatocellular carcinoma. Lancet 379: 1245-1255, 2012.

6. Lim KC, Chow PK, Allen JC, Chia GS, Lim M, Cheow PC, Chung AY, Ooi LL and Tan SB: Microvascular invasion is a better predictor of tumor recurrence and overall survival following surgical resection for hepatocellular carcinoma compared to the Milan criteria. Ann Surg 254: 108-113, 2011.

7. Du M, Chen L, Zhao J, Tian F, Zeng H, Tan Y, Sun H, Zhou J and Ji Y: Microvascular invasion (MVI) is a poorer prognostic predictor for small hepatocellular carcinoma. BMC Cancer 14: 38, 2014.

8. Rodríguez-Perálvarez M, Luong TV, Andreana L, Meyer T, Dhillon AP and Burroughs AK: A systematic review of microvascular invasion in hepatocellular carcinoma: Diagnostic and prognostic variability. Ann Surg Oncol 20: 325-339, 2013.

9. Roayaie S, Blume IN, Thung SN, Guido M, Fiel MI, Hiotis S, Labow DM, Llovet JM and Schwartz ME: A system of classifying microvascular invasion to predict outcome after resection in patients with hepatocellular carcinoma. Gastroenterology 137: $850-855,2009$

10. Hirokawa F, Hayashi M, Miyamoto Y, Asakuma M, Shimizu T, Komeda K, Inoue Y and Uchiyama K: Outcomes and predictors of microvascular invasion of solitary hepatocellular carcinoma. Hepatol Res 44: 846-853, 2014.

11. Chou CT, Chen RC, Lin WC, Ko CJ, Chen CB and Chen YL: Prediction of microvascular invasion of hepatocellular carcinoma: Preoperative CT and histopathologic correlation. AJR Am J Roentgenol 203: W253-W259, 2014.

12. Lee S, Kim SH, Lee JE, Sinn DH and Park CK: Preoperative gadoxetic acid-enhanced MRI for predicting microvascular invasion in patients with single hepatocellular carcinoma. J Hepatol 67: 526-534, 2017.

13. Xu P, Zeng M, Liu K, Shan Y, Xu C and Lin J: Microvascular invasion in small hepatocellular carcinoma: Is it predictable with preoperative diffusion-weighted imaging? J Gastroenterol Hepatol 29: 330-336, 2014.

14. Ahn SY, Lee JM, Joo I, Lee ES, Lee SJ, Cheon GJ, Han JK and Choi BI: Prediction of microvascular invasion of hepatocellular carcinoma using gadoxetic acid-enhanced MR and (18)F-FDG PET/CT. Abdom Imaging 40: 843-851, 2015.

15. Castellano G, Bonilha L, Li LM and Cendes F: Texture analysis of medical images. Clin Radiol 59: 1061-1069, 2004.

16. Scalco E and Rizzo G: Texture analysis of medical images for radiotherapy applications. Br J Radiol 90: 20160642, 2017. 
17. Canellas R, Burk KS, Parakh A and Sahani DV: Prediction of pancreatic neuroendocrine tumor grade based on CT features and texture analysis. AJR Am J Roentgenol 210: 341-346, 2018.

18. Bashir U, Siddique MM, Mclean E, Goh V and Cook GJ: Imaging heterogeneity in lung cancer: Techniques, applications, and challenges. AJR Am J Roentgenol 207: 534-543, 2016.

19. Giganti F, Antunes S, Salerno A, Ambrosi A, Marra P, Nicoletti R, Orsenigo E, Chiari D, Albarello L, Staudacher C, et al: Gastric cancer: Texture analysis from multidetector computed tomography as a potential preoperative prognostic biomarker. Eur Radiol 27: 1831-1839, 2017

20. Chamming's F, Ueno Y, Ferré R, Kao E, Jannot AS, Chong J, Omeroglu A, Mesurolle B, Reinhold C and Gallix B: Features from computerized texture analysis of breast cancers at pretreatment MR imaging are associated with response to neoadjuvant chemotherapy. Radiology 286: 412-420, 2018.

21. Nakajo M, Jinguji M, Nakajo M, Shinaji T, Nakabeppu Y, Fukukura Y and Yoshiura T: Texture analysis of FDG PET/CT for differentiating between FDG-avid benign and metastatic adrenal tumors: Efficacy of combining SUV and texture parameters. Abdom Radiol (NY) 42: 2882-2889, 2017.

22. Li Z, Mao Y, Huang W, Li H, Zhu J, Li W and Li B: Texture-based classification of different single liver lesion based on SPAIR T2W MRI images. BMC Med Imaging 17: 42, 2017.

23. Kloth C, Thaiss WM, Kärgel R, Grimmer R, Fritz J, Ioanoviciu SD, Ketelsen D, Nikolaou K and Horger M: Evaluation of texture analysis parameter for response prediction in patients with hepatocellular carcinoma undergoing drug-eluting bead transarterial chemoembolization (DEB-TACE) using biphasic contrast-enhanced CT image data: Correlation with liver perfusion CT. Acad Radiol 24: 1352-1363, 2017.

24. Gu Y, She Y, Xie D, Dai C, Ren Y, Fan Z, Zhu H, Sun X, Xie H, Jiang $G$ and Chen C: A texture analysis-based prediction model for lymph node metastasis in stage IA lung adenocarcinoma. Ann Thorac Surg 106: 214-220, 2018.

25. Obuchowski NA: Receiver operating characteristic curves and their use in radiology. Radiology 229: 3-8, 2003.

26. Pawlik TM, Delman KA, Vauthey JN, Nagorney DM, Ng IO, Ikai I, Yamaoka Y, Belghiti J, Lauwers GY, Poon RT and Abdalla EK: Tumor size predicts vascular invasion and histologic grade: Implications for selection of surgical treatment for hepatocellular carcinoma. Liver Transpl 11: 1086-1092, 2005.

27. Kim SJ, Lee KK and Kim DG: Tumor size predicts the biological behavior and influence of operative modalities in hepatocellular carcinoma. Hepatogastroenterology 57: 121-126, 2010.

28. Kaibori M, Ishizaki M, Matsui K and Kwon AH: Predictors of microvascular invasion before hepatectomy for hepatocellular carcinoma. J Surg Oncol 102: 462-468, 2010

29. Shirabe K, Toshima T, Kimura K, Yamashita Y, Ikeda T, Ikegami T, Yoshizumi T, Abe K, Aishima S and Maehara Y: New scoring system for prediction of microvascular invasion in patients with hepatocellular carcinoma. Liver Int 34: 937-941, 2014.
30. Lei Z, Li J, Wu D, Xia Y, Wang Q, Si A, Wang K, Wan X, Lau WY, Wu M and Shen F: Nomogram for preoperative estimation of microvascular invasion risk in hepatitis B virus-related hepatocellular carcinoma within the Milan criteria. JAMA Surg 151: 356-363, 2016

31. Giganti F, Marra P, Ambrosi A, Salerno A, Antunes S, Chiari D, Orsenigo E, Esposito A, Mazza E, Albarello L, et al: Pre-treatment MDCT-based texture analysis for therapy response prediction in gastric cancer: Comparison with tumour regression grade at final histology. Eur J Radiol 90: 129-137, 2017.

32. Zhou Y, He L, Huang Y, Chen S, Wu P, Ye W, Liu Z and Liang C: CT-based radiomics signature: A potential biomarker for preoperative prediction of early recurrence in hepatocellular carcinoma. Abdom Radiol (NY) 42: 1695-1704, 2017.

33. Liu S, Liu S, Ji C, Zheng H, Pan X, Zhang Y, Guan W, Chen L, Guan Y, Li W, et al: Application of CT texture analysis in predicting histopathological characteristics of gastric cancers. Eur Radiol 27: 4951-4959, 2017.

34. Lubner MG, Stabo N, Abel EJ, Del Rio AM and Pickhardt PJ: CT textural analysis of large primary renal cell carcinomas: Pretreatment tumor heterogeneity correlates with histologic findings and clinical outcomes. AJR Am J Roentgenol 207: 96-105, 2016.

35. Zhao WC, Fan LF, Yang N, Zhang HB, Chen BD and Yang GS: Preoperative predictors of microvascular invasion in multinodular hepatocellular carcinoma. Eur J Surg Oncol 39: 858-864, 2013.

36. Hasegawa K, Kokudo N, Imamura H, Matsuyama Y, Aoki T, Minagawa M, Sano K, Sugawara Y, Takayama $T$ and Makuuchi M: Prognostic impact of anatomic resection for hepatocellular carcinoma. Ann Surg 242: 252-259, 2005.

37. Ng F, Kozarski R, Ganeshan B and Goh V: Assessment of tumor heterogeneity by $\mathrm{CT}$ texture analysis: Can the largest cross-sectional area be used as an alternative to whole tumor analysis? Eur J Radiol 82: 342-348, 2013.

38. Xu Y, van Beek EJ, Hwanjo Y, Guo J, McLennan G and Hoffman EA: Computer-aided classification of interstitial lung diseases via MDCT: 3D adaptive multiple feature method (3D AMFM). Acad Radiol 13: 969-978, 2006.

39. Imura S, Teraoku H, Yoshikawa M, Ishikawa D, Yamada S, Saito Y, Iwahashi S, Ikemoto T, Morine Y and Shimada M: Potential predictive factors for microvascular invasion in hepatocellular carcinoma classified within the Milan criteria. Int J Clin Oncol 23: 98-103, 2018.

This work is licensed under a Creative Commons Attribution-NonCommercial-NoDerivatives 4.0 International (CC BY-NC-ND 4.0) License. 